Philosophie ANTIQUE

\section{Philosophie antique}

Problèmes, Renaissances, Usages

15 | 2015

Questions sur le scepticisme pyrrhonien

\title{
Le lieu controversé de l'économie antique
}

Entre oikos et polis

\section{Étienne Helmer}

\section{(2) OpenEdition}

Journals

Édition électronique

URL : https://journals.openedition.org/philosant/404

DOI : $10.4000 /$ philosant.404

ISSN : 2648-2789

Éditeur

Éditions Vrin

\section{Édition imprimée}

Date de publication : 24 novembre 2015

Pagination : 179-204

ISBN : 978-2-7574-1141-4

ISSN : 1634-4561

\section{Référence électronique}

Étienne Helmer, «Le lieu controversé de l'économie antique », Philosophie antique [En ligne], 15 | 2015, mis en ligne le 01 novembre 2018, consulté le 02 décembre 2022. URL : http://

journals.openedition.org/philosant/404 ; DOI : https://doi.org/10.4000/philosant.404

\section{(c) () 8 ()}

Creative Commons - Attribution - Pas d'Utilisation Commerciale - Pas de Modification 4.0 International - CC BY-NC-ND 4.0

https://creativecommons.org/licenses/by-nc-nd/4.0/ 


\title{
LE LIEU CONTROVERSÉ DE L'ÉCONOMIE ANTIQUE : ENTRE OIKOS ET POLIS
}

\author{
Étienne HELMER
}

Université de Porto Rico

RÉSUMÉ. Pour la plupart des exégètes modernes, la pensée économique grecque se trouve exclusivement dans les traités « oikonomiques », et son objet se limite à l'économie domestique. Pourtant, cette pensée économique offre également une dimension politique, présente aussi bien dans ces traités spécialisés que dans des textes politiques consacrés à l'organisation de la cité. Cette dimension politique de la pensée économique antique consiste en particulier à s'interroger sur la proximité et la différence entre l'oikos et la polis, et entre le domaine économique et le domaine politique. Après en avoir donné des exemples provenant de diverses traditions philosophiques antiques, je montrerai plus particulièrement que chez Xénophon et Platon, les rapports entre ces deux sphères se présentent sous deux formes principales : une similitude étroite ou une différence articulée.

SUMMARY. Against the common idea that the ancient Greek economic thought was limited to the study of the domestic economy, I argue it was also of a political nature. One of its main objects consisted in questioning the similarities and differences between the oikos and the polis, and between economics and politics. First, I will give examples of such a preoccupation excerpted from different Greek philosophical traditions. Second, I will show that in Xenophon's and Plato's works in particular, the relations between these two spheres took at least two different forms: they revealed to be a close similarity or a differentiated articulation. 



\section{Introduction}

Dans le sillage de la typologie wébérienne opposant l'homo ceconomicus moderne à l'homo politicus antique ${ }^{1}$, les penseurs politiques et les historiens de l'économie du siècle passé n'ont souvent vu dans les textes anciens consacrés aux pratiques et aux institutions économiques que des traités d'économie domestique dépourvus de tout enjeu politique ${ }^{2}$. Les historiens de l'Antiquité proposent certes aujourd'hui une vision beaucoup plus complexe et nuancée du monde économique antique, en montrant notamment que l'économie était aussi à certains égards l'affaire de la cité 3 . Mais, à de très rares exceptions près ${ }^{4}$, l'étude de la pensée économique antique n'a pas, pour sa part, bénéficié d'un semblable renouveau.

Quand on a parfois reconnu, à juste titre, que cette pensée pouvait aussi porter sur des phénomènes économiques débordant les limites de l'oikos, ce fut en suivant un schéma selon lequel l'oikonomia aurait été d'abord une pratique purement domestique - l'administration de l'oikos - qui aurait ensuite franchi les murs du foyer pour s'appliquer à la cités. Cette prétendue séquence chronologique semble aussi avoir été conçue comme une séquence logique comme si, de l'oikos à la polis, l'oikonomia passait naturellement du plus simple au plus complexe, du plus petit au plus grand. Une telle vision des choses, que refléterait l'évolution sémantique - in-

1. Voir Roth et Wittich 1978, vol. 2, p. 1354.

2. Voir par exemple Arendt 1961, ch. II et III.

3. Voir Reger 2005, p. 331-353 ; et surtout Scheidel, Morris et Saller 2007.

4. Notamment Leshem 2012, p. 201-209 ; et Leshem 2013, p. 43-61.

5. On trouve trace d'une telle façon de voir par exemple dans la façon dont C. Natali présente les différents sens du terme oikonomia selon leur «importance » - il ne dit pas comment il la détermine - chez les auteurs hellénistiques : partant du sens de « household management $»$, il passe à celui de « organized handling of wealth in the city », puis à celui de « good ordering of the cosmos » et enfin à celui de « organization of parts of a discourse 》 (Natali 1995, p. 95-128, en particulier p. 97-99). 
certaine - du terme oikonomia ${ }^{6}$, et qui fut entretenue par certains fondateurs de l'économie politique moderne ${ }^{7}$, a certes pu être suscitée par les textes anciens eux-mêmes. Par exemple, Xénophon fait dire à Socrate que l'administration réussie de l'oikos est propédeutique aux fonctions politiques, l'art du commandement étant un art unique dans tous les «genres d'activités, agriculture, politique, économie domestique, conduite de la guerre $\gg^{8}$. De même, l'Économique du Ps. Aristote (ou de Théophraste) fait se succéder un livre consacré à l'oikonomia au sens d'administration de l'oikos, puis un livre portant sur l'oikonomia au sens d'administration de divers territoires civiques. Dans les Politiques, Aristote commence lui aussi par analyser l'oikos avant d'en venir à la cité.

Cependant, cette interprétation qui prête aux Grecs un passage du domestique au politique sans solution de continuité, est aveugle au fait que les textes anciens consacrés à l'oikonomia, même quand ils soutiennent une position nette sur les rapports entre l'oikos et la polis ainsi qu'entre l'économique et le politique, le font toujours sur fond d'un questionnement implicite dont ils ne présentent, en général, que le résultat. C'est ce questionnement sous-jacent qu'il importe de restituer pour comprendre comment s'élabore la réflexion grecque sur l'oikonomia. Ainsi, après avoir montré que la question de l'identité ou de la différence entre économie et politique, et entre oikos et polis, est omniprésente dans les textes grecs consacrés à l'oikonomia, je montrerai que les relations entre ces deux sphères se présentent au moins sous deux formes différentes chez Xénophon et Platon notamment : une étroite similitude, et une différence articulée.

6. Descat 1988, p. 107, parle d'un « élargissement de l'oikonomia [s.e. c'est-à-dire du terme oikonomia] au domaine public » à partir du IV siècle. On verra plus bas que dans un article consacré à Thucydide (Descat 2010, p. 403-409, en particulier p. 407), le même auteur semble soutenir l'idée inverse : l'oikonomia aurait d'abord désigné la gestion politique des ressources publiques, avant de s'appliquer au domaine privé de l'oikos.

7. Les premières lignes de l'article «Économie politique » de Rousseau (1755) illustrent bien cette vision des choses : «ÉCONOMIE ou OECONOMIE (Morale et Politique) ce mot vient de oikos, maison, et de nomos, loi, et ne signifie originairement que le sage et légitime gouvernement de la maison, pour le bien commun de toute la famille. Le sens de ce terme a été dans la suite étendu au gouvernement de la grande famille, qui est l'État. » (Je souligne.)

8. Écon. XXI, 2. Voir aussi Écon. XIII, 5 et Mém. III, 6, 14 pour la fonction propédeutique de l'économique. 
Deux problèmes récurrents de la pensée économique antique : l'oikonomia est-elle similaire à la politique, l'oikos à la polis ?

L'interrogation sur les rapports entre l'oikos et la polis, ainsi qu'entre les arts respectifs, l'économique et la politique, permettant de diriger ces deux communautés, traverse la littérature économique antique. L'importance de cette question est perceptible dans la place liminaire qu'elle occupe dans la plupart de ces textes. En voici cinq exemples.

1) Quelques lignes après le début des Politiques, Aristote présente, pour la récuser, une position tenue à son époque sur ce sujet :

Quant à ceux qui pensent qu'être homme politique, roi, chef de famille,

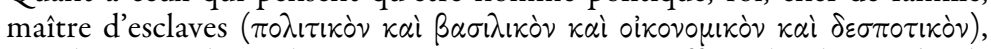
c'est la même chose, ils n'ont pas raison. C'est, en effet, selon le grand et le petit nombre, pensent-ils, que chacune de ces $<$ fonctions $>$ differre des autres, et non pas selon une <différence> spécifique : ainsi <quand on commanderait > à peu de gens, on serait maître, à plus de gens chef de famille, et à encore plus homme politique ou roi, comme s'il n'y avait aucune dif-

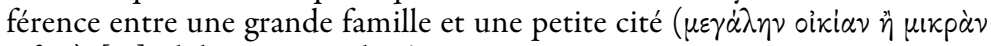
$\pi \dot{\partial} \lambda(v)$. [...] Eh bien, tout cela n'est pas vrai.

Politiques, I 1, 1252a8-16 (trad. P. Pellegrin).

Sans expliquer encore ce que sont et doivent être leurs liens, Aristote annonce ici sa thèse selon laquelle l'économique et la politique, comme l'oikos et la polis, sont deux choses distinctes. Il s'oppose ainsi à ceux qui ne voient qu'une différence de taille entre les deux. Qui est donc visé ?

2) On a coutume de croire qu'il s'agit de Xénophon et de Platon ${ }^{9}$. En ce qui concerne ce dernier, c'est le passage suivant, situé au début du Politique, qui serait la cible d'Aristote :

L'Étranger - Et celui qui possède cette science [i.e. la science royale], qu'il se trouve être souverain ou simple particulier, ne sera-t-il pas, du fait même de son art, tout à fait correct de l'appeler « royal »?

Socrate Le Jeune - Ce serait correct en effet.

L'Étranger - Or c'est la même chose pour l'intendant et le chef de famille

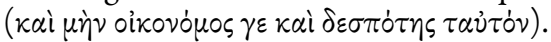

Socrate Le Jeune - Et comment !

L'Étranger - Alors quoi ! Pour ce qui est du commandement, y aura-t-il une différence entre la structure d'une grande résidence et le volume d'une

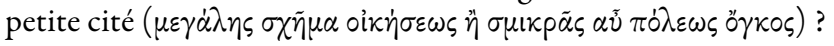

Socrate Le Jeune - Non, aucune.

9. Voir Pellegrin $1993^{2}$, p. 85 n. 2. Voir aussi Descat 1988, p. 108, selon qui l'idée qu'« il n'y a pas de différence de nature, mais seulement de dimension [...] entre les affaires du patrimoine et les affaires publiques [...] est une idée très chère au groupe socratique et à Platon, à laquelle s'opposera Aristote ». 
L'Étranger - En conséquence, comme on vient de l'examiner, il est clair qu'il existe une science unique qui concerne tout cela. Et cette science,

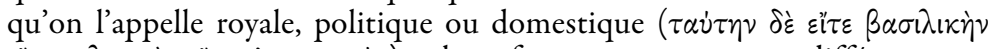

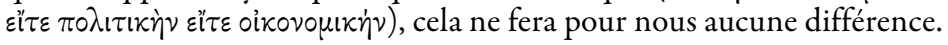

Socrate Le Jeune - Pourquoi cela en ferait-il une en effet ?

Politique, 259b2-c3 (trad. L. Brisson, J.-F. Pradeau modifiée).

On a cru lire dans ce passage le dernier mot de Platon sur l'identification de la cité et de l'oikos: ces deux communautés seraient selon lui identiques, à la différence de taille près, et l'économique et la politique seraient donc deux compétences semblables. Attribuer cette position à Platon est cependant difficilement tenable, pour un ensemble de raisons qu'il n'est pas possible de mentionner toutes $\mathrm{ici}^{10}$. Notons seulement que dans ce passage Platon n'emploie pas le terme oikos mais celui d'oikesis, qui désigne plus généralement un lieu de résidence, pas nécessairement domestique mais assurément pas encore politique au sens de lieu organisé selon les institutions de la polis ${ }^{11}$. Platon a beau mentionner conjointement la maison et la cité à plusieurs reprises dans ses Dialogues, rien ne permet de conclure que lui-même ou ses supposés porte-parole prennent à leur compte cette assimilation. Ce passage du Politique ne correspond qu’à une étape provisoire dans la recherche de la définition de l'homme politique et de son art, et ne permet aucunement de conclure, comme le confirmera la seconde section de cet article, que Platon identifie définitivement oikos et polis, économique et politique. Platon, comme ses contemporains, fait néanmoins de la question de l'identité ou de la différence entre économie et politique une question préliminaire fondamentale de sa philosophie politique.

3) C'est à juste titre, en revanche, que Xénophon est évoqué comme cible du texte d'Aristote par lequel nous avons commencé. La question de la nature respective des communautés domestique et civique, et des compétences requises pour bien les diriger, se trouve en effet formulée comme telle dans un passage des Mémorables (III 4, 1-12). À la différence des exemples précédents tirés d'Aristote et de Platon, ce passage n'apparaît toutefois pas au début de l'ouvrage, peut-être parce que celui-ci ne relève pas spécifiquement du logos oikonomikos, ou parce qu'il évoque l'oikonomia sans en faire pour autant un enjeu central de sa réflexion. Quoi qu'il en soit,

10. Pour une analyse détaillée de ce passage et sur le fait que Platon n'identifie pas l'oikos et la polis, voir Helmer 2010, p. 210-221.

11. LSJ s.v. Voir par exemple Aristote : «Le citoyen n'est pas citoyen par le fait d'habiter tel endroit, car des métèques et des esclaves partagent leur résidence ( $\tau \tilde{\eta} \varsigma$ oikn் $\sigma \varepsilon \omega \varsigma)$ avec lui », Politiques III, 1, 1275a8. La mention des métèques laisse penser que le terme oikesis ne désigne pas ici la maison. 
l'interrogation sur les similitudes et les différences entre l'économie domestique et la politique est suscitée par la surprise et la déception de Nichomachidès, soldat expérimenté, de voir les Athéniens lui préférer Antisthène pour assumer le poste de stratège. Selon Nichomachidès en effet, « Antisthène n'a jamais servi comme hoplite, n'a jamais rien fait de saillant dans la cavalerie, et ne sait rien qu'amasser de l'argent » (III 4, 2). Socrate, son interlocuteur, suggère alors que la compétence économique domestique, tout comme celle de chorège, est très pertinente pour faire un bon stratège, ce qui provoque l'incrédulité de Nichomachidès :

[6] - Tu dis donc, Socrate, que le même homme peut être à la fois bon chorège et bon stratège ? - Je dis qu'un homme qui, placé à la tête de quoi que ce soit, sait ce qu'il faut et se le procure, sera un excellent directeur,

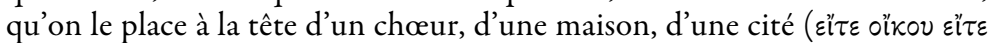
$\pi \dot{\lambda} \lambda \varepsilon \omega \varsigma)$, d'une armée. [7] Alors Nicomachidès : «Par Zeus, Socrate, je n'aurais jamais cru t'entendre dire que les bons administrateurs domestiques (oikovónol) peuvent être bons généraux. - Eh bien, examinons les actions des uns et des autres, et voyons si ce sont les mêmes ou si elles

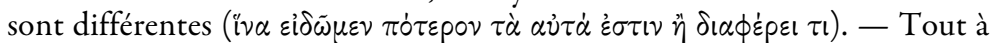
fait, dit-il.

Mémorables, III 4, 6-7 (trad. L.-A. Dorion modifiée).

On verra plus bas sur quelle base Xénophon rapproche étroitement la compétence économique et la politique, ainsi que la maison et la cité.

4) Dans l'Économique, le Ps. Aristote (ou Théophraste) est fidèle à Aristote. Comme lui, dès les premières lignes de son traité, il aborde la question de la différence et de la similitude entre économie et politique, entre oikos et polis :

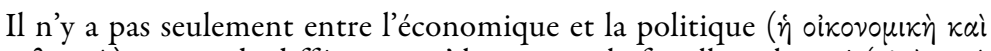

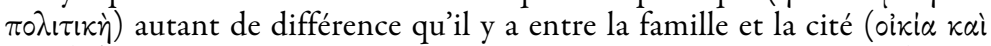
$\pi \dot{\partial} \lambda \iota \varsigma$ ) (car tels sont bien les objets respectifs de ces deux disciplines) mais encore celle-ci : la politique est l'affaire de beaucoup de chefs, l'économique d'un seul. Certains parmi les arts comportent des divisions : ce n'est pas au même qu'il appartient de produire et d'utiliser le produit, comme dans le cas de la lyre et de la flûte ; la politique, elle, a pour objet à la fois la constitution de la cité depuis l'origine, et son bon usage une fois constituée; aussi est-il évident qu'on peut en dire autant de l'économique, qui a pour objet l'acquisition de la maison et son usage.

Économique, I 1, 1343a1-9 (trad. A Wartelle modifiée).

Même si, comme chez Aristote, les deux premières lignes de ce passage laissent imaginer une différence de nature qui n'est pas encore précisée en- 
tre oikia et $p{ }^{1}{ }^{12}{ }^{12}$, la fidélité de Théophraste à son maître s'arrête là. Car contrairement à Aristote, il donne à la différence quantitative ou numérique une importance de premier plan en évoquant le nombre des chefs respectifs de la cité et de la maison. Cette différence quantitative fait fond sur une identité fonctionnelle - l'art économique et l'art politique sont tous deux à la fois des arts d'acquisition et d'usage - qu'Aristote récuse aussi.

5) Même les auteurs qui laissent manifestement la politique de côté dans leurs développements sur l'économie y consacrent néanmoins quelques mots en soulevant précisément la question de leurs rapports. C'est le cas de Philodème de Gadara. Conformément au courant épicurien qui, sans rejeter la politique, lui octroie toutefois une place marginale en comparaison de l'éthique ${ }^{13}$, Philodème se contente de l'évoquer dans sa critique de l'Économique de Théophraste (ou du Ps. Aristote). Cette critique occupe là encore une place liminaire dans le traité, puisqu'elle se situe quelques paragraphes après le début du chapitre IX des Vices, qui forme par luimême un traité sur l'oikonomia :

Superflue assurément son [i.e. Théophraste] entrée en matière, car cela n'intéresse en rien l'économie qu' « elle differe de la politique », même s'il est faux de dire que, dans l'absolu, « la politique » n'est pas « l'affaire d'un chef unique » tandis que dans l'absolu, « l'économie l'est », et qu'il n'y a jamais d'analogie entre les deux.

Les Vices, IX (trad. D. Delattre et V. Tsouna modifiée, p. 600).

Pour rapide que soit l'allusion, Philodème semble estimer que ce point est suffisamment important pour faire l'objet de sa critique, comme si, là encore, il fallait préciser d'emblée le rapport entre économique et politique $^{14}$.

12. Une suggestion sur cette différence est faite peu après par le Ps. Aristote, lorsqu'il

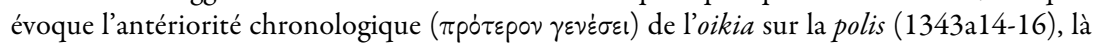

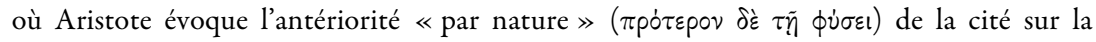
maison (Politiques, I 1, 1253a18-19). On pourrait imaginer que l'antériorité «par nature » ou antériorité «logique » n’implique pas nécessairement l'antériorité chronologique, et qu'Aristote pourrait donc être d'accord avec le Ps. Aristote sur l'antériorité chronologique de la maison sur la cité. Mais rien ne permet ici d'en décider.

13. Voir Brown 2009, p. 179-196; et Morel 2007, p. 167-186.

14. Philodème lisait sans doute un texte distinct du nôtre, car dans la version de l'Économique dont nous disposons, Théophraste (ou le Ps. Aristote) ne dit pas qu'il n'y a pas d'analogie entre économique et politique. On trouve une autre allusion à la politique chez Philodème quand il évoque de riches personnages notoires - « Gellias de Sicile, Scopas de Thessalie et les Athéniens Cimon et Nicias »- apparemment mentionnés par Métrodore dans son livre La Richesse, et dont il semble critiquer la violence avec laquelle ils ont acquis leur richesse (Les Vices, IX, p. 611-612). 
La plupart des auteurs grecs qui s'interrogent sur l'oikonomia font donc de la question de la différence ou de la similitude entre l'économique et le politique, entre cité et oikos, un passage incontournable de leur réflexion. Comment donc conçoivent-ils leurs rapports ?

Économie et politique, oikos et polis: le même ou l'autre

Ni préoccupés uniquement de la cité, ni confinés théoriquement dans l'espace clos de l'économie domestique dès qu'ils s'interrogent sur l'oikonomia et sur l'économie en général, les auteurs grecs des périodes classique et hellénistique se montrent au contraire soucieux, dans leurs ouvrages «oikonomiques » comme dans une grande partie de leurs traités politiques, de penser les relations entre l'économique et le politique, entre l'oikos et la polis. On peut distinguer, dans les deux cas, deux relations principales entre les deux termes : l'étroite similitude ou la différence articulée.

Callicratidas et Xénophon

ou l'étroite similitude de l'oikos et de la polis, de l'économique et du politique

Un fragment du pythagoricien Callicratidas intitulé «Sur le bonheur de l'oikos », pose une relation d'analogie entre, d'un côté, la cité et l'oikos, et, de l'autre, le cosmos:

C'est selon le même principe que sont organisées, dans les affaires humaines, la maison et la cité, et dans les affaires divines, le cosmos; car la maison et la cité sont des imitations analogiques de l'organisation du cosmos.

Sur le bonheur de loikos, Stob. 4.28 .17 p. 685 He. (Mullach 2 p. 31) $=$ Thesleff p. 105 (ma traduction ${ }^{15}$ )

L'adjectif «analogique » employé dans ce passage se justifie par le fait que la ressemblance ou la similitude entre la maison et la cité se fonde sur un modèle commun : celui de l'organisation de l'univers, dont elles « sont des imitations, conformément à la proportion ${ }^{16}$. Quelques lignes avant ce passage, Callicratidas a en effet montré que l'univers était comme un ensemble harmonieux d'éléments disparates, à l'image d'un chœur musical ou du plan de construction d'un navire : l'unité harmonieuse de chacun de ces ensembles provient de leur finalité respective, tournée vers ce qui est le mieux pour chacun d'eux. Mais la supériorité de l'harmonie de l'univers tient au fait qu'elle est d'origine divine, donc la meilleure, et qu'elle est par-

15. Les fragments de Callicratidas sont cités d'après leur édition en grec dans Thesleff 1965, p. 102-107. Je les traduis. Les références indiquées sont celles données par H. Thesleff, auxquelles j'ajoute le numéro de la page de son édition.

16. Delatte 1922, p. 164. 
faitement «politique » $(\pi \circ \lambda \imath \tau \iota \kappa \dot{\alpha})$ au sens où elle est en vue du tout du monde, de toutes ses parties, aussi bien de celles qui gouvernent que de celles qui sont gouvernées. Selon un schéma de pensée fréquent chez les pythagoriciens, l'oikos et la polis sont des adaptations de ce modèle ${ }^{17}$, que Callicratidas exploite pour présenter la relation conjugale dans l'oikos sur un mode similaire à ce qui se passe (ou doit se passer) dans la cité : le mari doit commander à son épouse non pas uniquement dans son intérêt à lui ni seulement dans le sien à elle, mais dans l'intérêt de leur communauté. La suite du texte évoque une condition de possibilité de cette communauté domestique : l'égale puissance économique et sociale entre les époux, sans laquelle le mari sera en conflit avec sa femme si elle est plus riche que lui elle cherchant à commander, lui refusant d'obéir -, ou sans laquelle la réputation du mari et de sa famille sera assombrie si sa femme est de moindre extraction que lui (Stob. 4.28 .18 p. 687 He. (Mullach 2 p. 30) $=$ Thesleff p. 106).

Ce texte ne permet pas cependant de tirer de plus amples conclusions sur le rapprochement entre l'oikos et la polis. Tout au plus peut-on dire que Callicratidas affirme la similitude de la finalité générale de l'oikos et de la polis, sans que cela implique nécessairement une identité de leurs finalités particulières respectives, procurer le nécessaire dans un cas, gouverner les hommes dans l'autre. C'est vers l'œuvre de Xénophon qu'il faut se tourner pour trouver une représentation plus précise de l'étroite similitude entre les champs politique et économique. Cette similitude porte plus précisément sur cinq points, qui justifient que l'administration domestique soit présentée par le Socrate de Xénophon comme propédeutique à la politique.

1) Pour le Socrate de Xénophon comme pour son Ischomaque, maison et cité ne diffèrent qu'en degré, pas en nature. La maison est une cité miniature ${ }^{18}$, comme le souligne le passage suivant :

[12] Socrate - Ne méprise donc pas, Nicomachidès, les hommes qui s'oc-

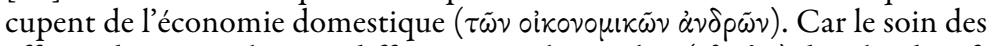
affaires des particuliers ne differe que par le nombre $(\pi \lambda \dot{\eta} \theta \varepsilon l)$ de celui des affaires publiques : tous les autres points sont similaires $(\tau \dot{\alpha} \delta \dot{\varepsilon} \ddot{\alpha} \lambda \lambda \alpha \pi \alpha \rho \alpha-$ $\pi \lambda \dot{\eta} \sigma \iota \alpha$ है $\chi \varepsilon 1)$.

Mémorables III, 4, 12 (trad. L.-A. Dorion modifiée).

2) Que la maison soit très semblable à la cité est confirmé par le fait que pour enseigner la justice au personnel de sa maison, en particulier au

17. Un fragment de Théon de Smyrne (Exp. p. 12, 18, H), cité par Delatte 1922, p. 167, explique que l'ordre des éléments en musique est le gouvernement «aristocratique » du tout; dans l'univers, cet ordre est l'harmonie, dans la cité la bonne législation, et dans la maison la sagesse.

18. Et non la métaphore de la cité, comme le soutient Plácido Suárez 2001, p. 20. 
responsable de l'agriculture, Ischomaque fait des emprunts « tantôt aux lois de Dracon, tantôt aux lois de Solon » (Écon. XIV 4), ainsi qu'à celles du Grand Roi (Écon. XIV 6), et qu'il invite sa femme à penser sa fonction domestique par analogie avec le gardien des lois dans la cité :

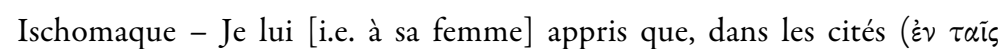
$\pi \dot{\lambda} \lambda \varepsilon \sigma \iota \nu)$ soumises à de bonnes lois, les citoyens ne croient pas suffisant de se donner de bonnes lois; ils choisissent comme gardiens de ces lois des hommes qui, sentinelles vigilantes, approuvent ceux qui les observent et punissent ceux qui les transgressent. [15] Je recommandai à ma femme de se considérer comme gardienne des lois dans notre maison ( $\dot{\varepsilon} \nu \tau \tilde{\eta}$ oiki $\alpha$ ) [...].

Économique, IX, 14-15 (trad. E. Talbot modifiée).

Ce passage ne permet pas de conclure que la maison et la cité sont totalement identiques, mais il indique clairement qu'elles reposent sur des principes directeurs similaires, d'origine politique. Cette similitude explique que la direction des affaires politiques et économiques soit l'affaire des mêmes hommes, non de spécialistes différents, comme Socrate l'explique à Nicomachidès dans la suite immédiate du passage des Mémorables cité dans la section précédente ${ }^{19}$.

3) L'oikos et la polis ont une finalité commune: la croissance. Commençons par l'oikos. La croissance est mentionnée dans la définition de l'oikonomia à laquelle aboutissent Socrate et Critobule :

[4] Eh bien, dit Socrate, le nom d'économie (oixovoui $\alpha$ ) nous a paru être

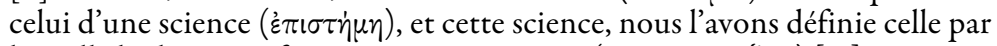

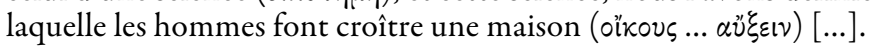

Économique VI, 4 (trad. E. Talbot modifiée).

On retrouve cet élément dans les propos d'Ischomaque à sa femme :

[15] [...] il est du devoir d'un homme et d'une femme sages de maintenir leur avoir dans le meilleur état possible, et de l'accroître $(\pi \lambda \varepsilon \tilde{\tau} \sigma \tau \alpha)$ autant que possible par des moyens honnêtes et justes.

Économique VII, 15 (trad. E. Talbot modifiée).

À sa femme qui lui demande alors comment elle peut contribuer à cette

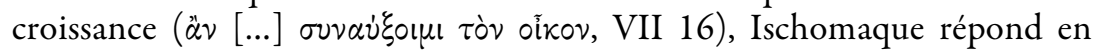

19. « Socrate: [...] et l'essentiel, c'est que les unes et les autres [i.e. les affaires domestiques et les affaires politiques] ne peuvent se traiter que par des hommes, et que ce ne sont pas tels hommes qui font les affaires privées ( $\tau \dot{\alpha}$ i $\delta \hat{\imath} \alpha$ ), et tels autres les affaires communes ( $\tau \dot{\alpha}$ kotvd), que ceux qui dirigent les affaires communes n'emploient pas certains hommes, et cer-

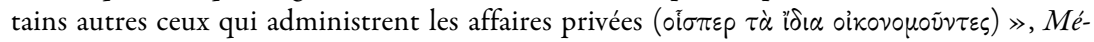
morables, III 4, 12. 
deux temps. Il lui explique d'abord que la nature a réparti les tâches entre les sexes (VII 17-32), et il lui fait voir ensuite, parmi les fonctions qui lui reviennent, toute l'importance de la conservation des biens acquis (VII 33, 39-40), notamment par le rangement ordonné de tout ce qui entre dans la maison (VIII 1-IX, 10).

Concernant la cité, Socrate explique à l'ambitieux Glaucon en quoi consiste la compétence politique dans les termes suivants :

[2] [...] Glaucon, dit-il, tu t'es donc mis dans la tête de gouverner notre cité ? - Mais oui, Socrate. - Par Zeus, c'est le plus beau des projets qu'un homme puisse former : car il est clair que, si tu parviens à ton but, tu seras capable d'obtenir tout ce que tu désireras, de servir tes amis, de faire briller la maison paternelle, d'agrandir ta patrie ( $\alpha \dot{\zeta} \xi \dot{\eta} \sigma \varepsilon ı \varsigma \delta \dot{\varepsilon} \tau \dot{\eta} \nu \pi \alpha \tau p i \delta \alpha)[\ldots]$.

Mémorables III, 6, 2 (trad. L.-A. Dorion modifiée).

La dernière expression, parallèle à ce qui a été dit de la maison dans les extraits de l'Économique cités juste avant, confirme que l'oikia et la polis visent un même but. Mais comment augmenter la cité ? Il faut se tourner vers l'ouvrage connu sous le titre Les Revenus pour voir quels moyens Xénophon suggère pour parvenir à cet objectif. Dans cet ouvrage, il propose de remédier à la pauvreté qui sévit à Athènes en accroissant ses finances par différents moyens, mais toujours à partir des ressources propres de la cité ( $\dot{\varepsilon} \kappa \tau \tilde{\eta} \varsigma \dot{\varepsilon} \alpha \nu \tau \tilde{\omega} \nu$, Revenus, I 1). À lui seul, ce texte ne permet certes pas d'affirmer que, pour Xénophon, la croissance de la cité est l'unique but de la politique, bien que d'autres textes semblent aller en ce sens ${ }^{20}$. Mais rapportée à la fois à la citation précédente des Mémorables, et à la thèse de la similitude étroite de la cité et de la maison, la recherche des moyens d'accroître les revenus de la cité est tout à fait cohérente avec cette recherche de croissance qu'on a observée au niveau de l'oikos. Xénophon montre ainsi que «notre pays donne par nature de forts revenus » (ö $\tau\llcorner\dot{\eta}$

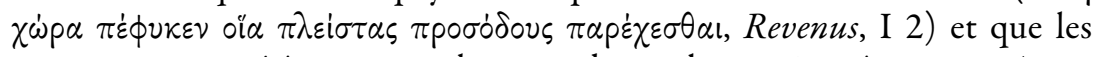
commerçants métèques en plus grand nombre, sujets à un impôt et exempts d'obligations militaires, « augmenteraient les revenus » de la cité

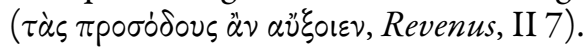

4) Le modèle comptable présenté par Xénophon pour la maison et la cité est en grande partie le même : dans les deux cas, il s'agit de trouver l'activité la plus lucrative ( $\kappa \varepsilon p \delta \alpha \lambda \varepsilon \omega \tau \dot{\alpha} \tau \eta, \kappa \varepsilon p \delta \alpha \lambda \varepsilon \dot{\omega} \tau \varepsilon p o v$, Revenus, III $1 ; \mathrm{V} 11$ ), le gain étant calculé soit en valeur absolue, soit de façon relative grâce à une diminution des dépenses, ces dernières devant être prises dans les deux cas

20. Par exemple dans l'Anabase, où Xénophon parle de lui à la troisième personne, il écrit « qu'il serait glorieux pour lui d'accroître le territoire et la puissance de l'Hellade par la

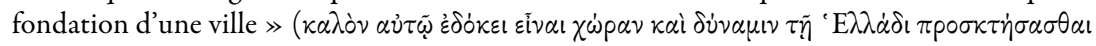

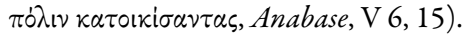


non sur le capital mais sur les excédents dégagés ${ }^{21}$. La croissance évoquée dans le point précédent est donc à entendre à la fois comme croissance brute, mais aussi comme croissance relative obtenue par la maîtrise ou la baisse des dépenses.

Commençons par la cité. Proposant d'inciter les marchands à venir commercer à Athènes en accordant des distinctions « à ceux qui parâ̂traient utiles à la cité par l'importance de leurs vaisseaux et de leurs cargaisons » (Revenus, III 4), Xénophon en attend une croissance mécanique du volume d'affaires traitées à Athènes et de leurs retombées économiques, retombées d'autant plus grandes que « cette augmentation de revenus ne

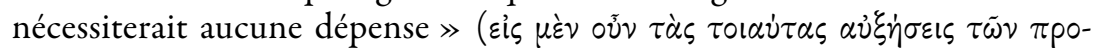

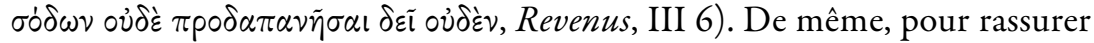
ceux qui pourraient juger économiquement irréalisable son projet d'exploitation des mines d'argent, Xénophon préconise une méthode progressive d'investissement :

[36] [...] il est plus avantageux de procéder par fractions ( $\kappa \alpha \tau \dot{\alpha} \mu \dot{\varepsilon} \rho \circ \varsigma)$ que d'entreprendre tout ensemble $(\ddot{\alpha} \mu \alpha \pi \dot{\alpha} \nu \tau \alpha)$. En construisant beaucoup à la fois, on dépense plus, et on fait moins bien qu'en opérant successivement ; en cherchant partout des esclaves, on est forcé de les acheter moins bons et plus chers ; [37] tandis qu'en opérant selon ses moyens, si une entreprise est bien conçue, on la suit ; [38] si on se trompe, on l'abandonne. D'ailleurs, pour exécuter tout ensemble, il faut avoir des moyens pour tout, au lieu qu'en terminant ceci et en ajournant cela, la rentrée du revenu vient en aide à ce qui reste à faire.

Revenus, IV 36-38 (trad. E. Talbot modifiée).

Au contrôle des dépenses s'ajoute, à la fin de ce passage, l'idée que les revenus dégagés peuvent devenir à leur tour productifs, sans qu'il soit nécessaire pour cela de toucher au capital. Enfin, à propos de la paix comme condition du renouveau financier d'Athènes, Xénophon y voit « une occasion pour notre cité de regagner l'affection des Grecs sans peine, sans

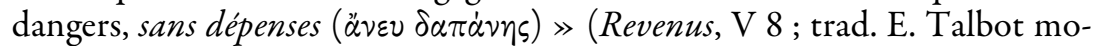
difiée ; je souligne).

Ce contrôle des dépenses vaut également pour l'oikos. Critobule comprend bien que pour Socrate, le moyen de devenir riche, « c'est de dégager

21. Cette même rationalité comptable semble avoir été celle de Périclès, qui l'aurait appliquée à la cité puis à sa propre maison. Voir Descat 2010, p. 403-409, en particulier p. 405. On trouve aussi chez le Ps. Aristote deux remarques générales sur le contrôle des dépenses tant au niveau de l'économie domestique, selon la taille de l'exploitation (Écon. I 6, 1345a17-18), que de l'économie satrapique (Écon. II, 7, 1346a21-24). Voir aussi chez Pla-

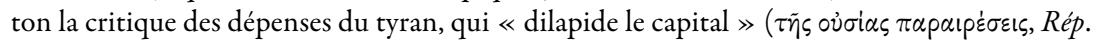
IX, 573e1). 


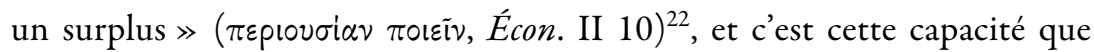

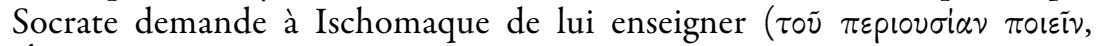
Écon. XI 13). Deux méthodes sont mises en concurrence : l'accumulation des biens, qui attire Critobule, et le contrôle des dépenses fondé sur la maîtrise des appétits que Socrate préconise. C'est ce même contrôle des dépenses qu'Ischomaque attend de sa femme :

[il faudra] [36] recevoir ce que l'on apportera, distribuer ce que l'on devra dépenser, penser d'avance à ce qui devra être mis de côté, et veiller à ne pas faire pour un mois la dépense prévue pour une année.

Économique VII, 36 (trad. P. Chantraine).

Le lien entre dégager un surplus et contrôler les dépenses est explicite dans la citation suivante :

Quand tous les frais sont complètement prélevés sur le patrimoine, et que les travaux ne sont pas conduits de manière à couvrir la dépense, on ne doit

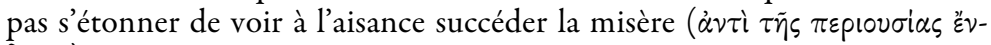
$\delta \varepsilon(\alpha \nu)$.

Économique XX, 21 (trad. E. Talbot)

Polis ou oikos, il s'agit donc de croître, avec dans les deux cas, la préoccupation que cet accroissement se fasse par des moyens socialement et moralement légitimes. En ce qui concerne l'oikos, Socrate analyse en effet ce

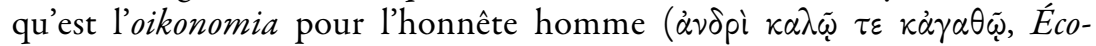
nomique, VI 8), c'est-à-dire un homme respectueux des valeurs traditionnelles, morales ou civiques. De même Ischomaque explique à sa femme que

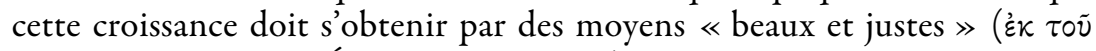

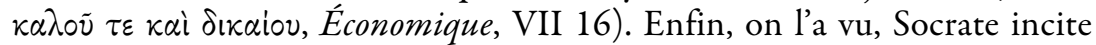
Critobule, sur l'exemple d'Ischomaque, à s'enrichir moins par l'accumulation sans limite que par le contrôle de ses propres appétits. Quant à la cité, Xénophon souligne que les procédés qu'il suggère pour augmenter les revenus de la cité devront être approuvés par les dieux dans les sanctuaires de Dodone et de Delphes (Revenus, VI 1-2).

5) C'est la terre et son «entretien » qui assurent chez Xénophon le lien matériel et fonctionnel entre l'économie et la politique, la terre étant à la fois le support de la production agricole et la base matérielle et symbolique du territoire dont il faut garantir l'intégrité. Dans la large section de l'Économique qu'il consacre à l'éloge de l'agriculture (V 1-17), Socrate commence par ses bienfaits «oikonomiques »: outre qu'elle est une source d'agrément et qu'elle fournit à l'homme libre l'occasion de développer toutes

22. « Dégager un surplus » plutôt que «faire des économies 》 (P. Chantraine, E. Talbot), ce qui serait plutôt le moyen de dégager ce surplus. 
ses facultés, elle est aussi « un moyen d'accroître sa maison 》 (oỉkov aủ$\xi \eta \sigma \iota$, V 1). Mais très vite, Socrate passe aux avantages militaires de cette pratique domestique de l'agriculture : «si l'on veut servir la cité dans la cavalerie, rien de plus capable que l'agriculture d'aider à nourrir le cheval; si l'on veut servir dans l'infanterie, elle rend le corps vigoureux » (V 5). Le travail agricole est, en somme, une excellente préparation militaire. Il développe les qualités physiques de ceux qui cultivent la terre comme de ceux qui surveillent les travaux des champs: il les rend endurants, forts et vigoureux (V 4), et leur apprend à « courir, à lancer le javelot, à sauter » (V 8). Il développe aussi chez eux des qualités morales, à commencer par le souci de ce qui est à soi, sur lequel la politique pourra compter pour la défense du territoire. Socrate signale en effet que l'un des multiples mérites de « la terre [est d'] encourage[r] aussi les cultivateurs à défendre leur pays les armes à la main, par ce fait même que ses productions sont offertes à qui veut, et la proie du plus fort » (V 7). Surtout, l'agriculture est une école de justice, car « la terre, étant une divinité, enseigne aussi la justice $»\left(\mathrm{~V} \mathrm{12} 2^{23}\right)$. La définition de cette valeur, précisée immédiatement après, renvoie à l'idée de réciprocité : « c'est à ceux qui lui témoignent le plus d'égards que la terre accorde en échange ( $\dot{\nu} \nu \tau \iota \pi \circ \varepsilon \tilde{\imath})$ le plus de biens » (V 12), ce dont Socrate a donné divers exemples peu auparavant ${ }^{24}$.

Ce rapide portrait des avantages de l'agriculture explique pourquoi la politique du Roi des Perses consiste à se « préoccuper de l'agriculture et de l'art de la guerre avec une égale ardeur » (IV 4 ; même idée en IV 12). Si l'agriculture domestique, on vient de le voir, sert l'art militaire, l'art militaire sert lui aussi l'agriculture. Les militaires, hiérarchiquement organisés, doivent en effet assurer l'intégrité du territoire face à un éventuel ennemi extérieur, pour que le travail des champs, supervisé par une administration civile, soit possible et pour que, outre les fruits qu'il produit, le tribut auquel il donne lieu puisse être prélevé (IV 9-11) ${ }^{25}$.

Outre leur complémentarité, l'agriculture et l'art militaire reposent sur une même conception du pouvoir. Le chef militaire et l'intendant des travaux agricoles doivent tous deux savoir commander aux hommes (V 14) en les rendant ardents au travail et obéissants par un système de récompenses, de punitions et d'encouragements (V 15-16) : ouvriers agricoles dans un

23. Même idée chez le Ps. Aristote : «l'agriculture [est] conforme à la justice », Écon. I 2, 1343a28.

24. « En échange des services qu'ils reçoivent de l'agriculture, chiens et chevaux de leur côté rendent service à la ferme ( $(\dot{\alpha} \nu \tau \omega \phi \varepsilon \lambda \circ \tilde{v} \sigma \mathrm{l}) \gg(\mathrm{V}$ 6); « Quel art paie mieux de retour $(\dot{\alpha} \nu$ $\tau \imath \chi \alpha p i \zeta \varepsilon \tau \alpha \iota)$ ceux qui le pratiquent ? » Voir aussi Cyropédie, VIII 3, 38 : «Un lopin de terre respecte plus que tout la justice : il rend bien et justement la semence qu'il a reçue. »

25. Le tribut agricole est mentionné aussi par le Ps. Aristote à propos de «l'économie satrapique », Écon. II 1, 1346a1-2. 
cas, soldats dans l'autre, les vertus des premiers les rendant aptes, si nécessaire, à tenir le rôle des seconds. Cette idée rejoint celle du texte cité plus haut en note dans la section 2), où Socrate explique que ce sont les mêmes hommes qui sont compétents pour la politique et pour l'économique (Mémorables III 4, 12).

Ces passages signalent combien l'agriculture, loin d'être seulement une technique tournée vers des fins économiques, est investie par Xénophon d'une fonction politique qui n'est pas seulement symbolique mais, pourrait-on dire, effective ou opérante. C'est presque sans distance que le politique se réalise ici dans et par l'économique, ce dont Cyrus le Jeune est, pour Xénophon, la parfaite incarnation. L'agriculture n'est certes pas le seul art entrant en jeu dans l'économie ${ }^{26}$, mais c'est « la mère et la nourrice des autres arts » (V 17) en raison du double rôle, économique et militaropolitique, de la terre.

Le modèle politique sur lequel se fonde cette étroite similitude entre oikos et polis est celui de l'empire perse, Xénophon ne cachant pas son admiration pour Cyrus le Jeune ${ }^{27}$. Or c'est précisément ce modèle, orienté vers la croissance et fondé sur un empire militaire conquérant ${ }^{28}$, que contestent autant Aristote que Platon pour des raisons éthiques et politiques. C'est pourquoi, même si tous deux accordent également à l'économique un rôle central dans la réalisation du politique, leurs propositions économiques et leur conception de l'oikos sont très distinctes de celles de Xénophon : il leur est inconcevable de rapprocher trop étroitement maison et cité, économie et politique.

Platon ou la différence articulée entre l'économique et le politique, entre l'oikos et la polis

C'est sans doute au livre I des Politiques d'Aristote que l'articulation entre l'oikos et la polis, et entre l'économique et le politique, est la plus ma-

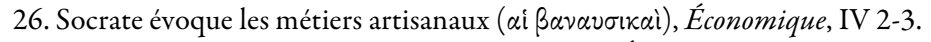

27. Voir Descat 1988, p. 118. Voir chez Xénophon Écon. IV 4.

28. Il y a une tension évidente chez Xénophon entre, d'un côté, l'affirmation qu'on trouve à la fin des Revenus (V 11-13) que la paix, bien mieux que la guerre, est nécessaire pour rendre possible l'accroissement des revenus par tous les moyens proposés dans cet ouvrage, et, de l'autre, la nécessité de croître qu'il assigne à loikos et à la polis dans l'Économique et dont l'offensive guerrière semble fournir le modèle et le moyen le plus propice (ce que signale assez nettement l'éloge de Cyrus le Jeune par Socrate, sans même parler de la $C y$ ropédie). La solution de cette difficulté est peut-être que, pour Xénophon, la guerre est en effet le meilleur moyen de croître quand le rapport de forces est favorable, sans quoi il faut évidemment lui préférer la paix, moins pour des raisons politiques que d'efficacité économique (dégager un surplus ou faire des économies). C'est ce que suggère un passage des Mémorables (III 6, 7-8). 
nifeste et la plus claire, parce que les niveaux d'intégration sociale et politique représentés par chacune de ces institutions sont nettement délimités. Contribue à cette délimitation le fait que dans ce même ouvrage, la fonction économique des citoyens dans la cité la meilleure n'est pas un critère légitime de citoyenneté, c'est même un obstacle à son exercice. Car « on n'est pas susceptible de pratiquer la vertu quand on mène une vie

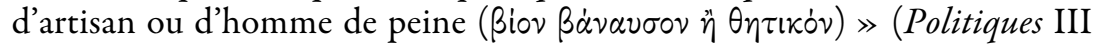
5, 1278a20), même si de fait, comme Aristote le reconnaît, il est fréquent que les citoyens travaillent : dans ce cas, il faut dire que l'excellence du citoyen est réservée à « ceux qui sont affranchis des tâches indispensables 》 (1278a10-11). Certes, la distinction des plans économique et politique semble s'estomper si l'on songe qu'Aristote distingue les oikoi «politiques », c'est-à-dire ceux qui, tant par leur agencement humain et matériel que par leur dimension morale, sont des conditions de possibilité de la polis, des oikoi «non politiques » qui sont soit tyranniques, soit isolés et non intégrés dans des formes supérieures de communauté, comme chez les $\mathrm{Cy}$ clopes d'Homère par exemple ${ }^{29}$. Mais les oikoi dits «politiques » le sont uniquement au sens où ils assurent la formation morale de leurs membres, et où la possession de la terre et le travail des esclaves rendent possible le loisir du propriétaire ${ }^{30}$. Ils sont comme la condition nécessaire mais non suffisante du politique, sans que l'exercice de fonctions économiques soit un facteur direct de réalisation politique.

Les choses sont différentes avec Platon, qui donne à la sphère économique un rôle beaucoup plus central qu'Aristote dans la réalisation du politique, sans toutefois jamais confondre l'oikos et la polis, ni l'économique et le politique. C'est ce qui apparaît dans la République et, plus précisément, dans deux passages du Politique.

\section{1) La République}

La République présente la différence entre la sphère économique et la sphère politique comme une différence entre deux modes de réalisation du

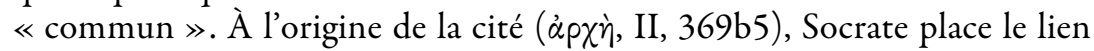
et les fonctions économiques nées de la nécessité où les individus se trouvent de satisfaire « beaucoup de besoins » (369b-c). Incapables d'y pourvoir par eux-mêmes, ils sont donc forcés de s'assembler. Mais Socrate place cette réunion d'individus sous le signe d'une forte tension entre le particulier et le commun. En effet, d'un côté, chacun accepte d'entrer dans un cycle d'échanges où il donne et reçoit « parce qu'il croit que c'est mieux

29. Sur ces différentes sortes d'oikoi non politiques, voir Brendan Nagle 2006, p. 135151.

30. Brendan Nagle 2006, p. 122. 


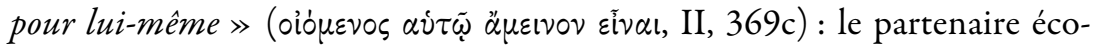
nomique n'est que l'instrument d'un besoin propre, l'accent étant donc mis ici sur le bien particulier ou privé. D'un autre côté, à l'échelle de la cité, l'organisation spontanée de l'économie est telle que chacun destine le

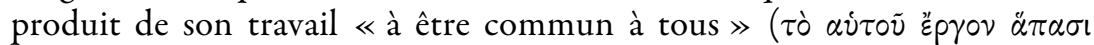

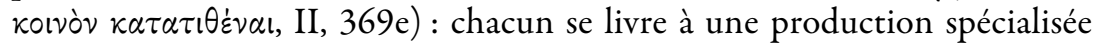
dont les fruits sont « mis en commun » au sens où ils seront échangés contre les produits d'une autre spécialité technique. Dans le même sens, Socrate rappelle peu après que le commerce a pour fonction de rendre pos-

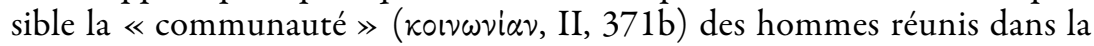
cité pour pourvoir à leurs besoins.

Un tel mélange de mise en commun et d'intérêt particulier ou privé rend la sphère politique instable, l'intérêt particulier tendant sans cesse à prévaloir sur le commun sous l'effet de la tendance des appétits à se multiplier et à devenir insatiables (II, 372e-373e). Plus tard dans le dialogue, au moment d'analyser la dégradation des régimes à partir de celui des philosophes-rois, Socrate souligne en effet que la cité se détériore dès que ses gardiens commencent à se comporter comme des oikonomoi préoccupés surtout, ou exclusivement, de leurs biens privés (VIII, 547a-c) au détriment $\mathrm{du}$ bien commun. Chaque étape de cette dégradation est marquée par l'emprise grandissante de l'intérêt particulier, qu'il concerne des groupes sociaux ou des individus, et de la dissension croissante qu'il provoque dans la cité. Par exemple, les timocrates valorisent les honneurs en public, mais en privé, ils « honoreront sans réserve, sous le couvert de l'ombre, l'or et l'argent, car ils posséderont des dépôts et des coffres-forts qui leur appartiendront en propre, où ils les placeront pour les cacher, et par ailleurs des enceintes autour des habitations, qui en feront des sortes de nids privés [...] » (VIII, 548a). En démocratie, chacun entend par liberté la satisfaction de son plaisir du moment (VIII, 561b), donc particulier, ce qui se traduit par le fait que dans ce régime «tout le monde recherche la ri-

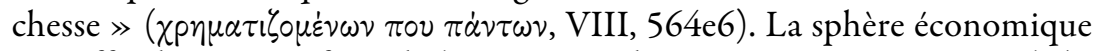
ne suffit donc pas à faire de la cité un ordre vraiment commun : en l'absence de tout organe de régulation extra-économique ou supra-économique, elle se transforme en instrument de l'intérêt particulier, donc en source de conflit.

Pour prévenir cette tendance, l'essentiel de la tâche politique telle qu'elle est présentée dans la République consiste à faire de la cité une véritable communauté. Pour y parvenir, Socrate propose deux mesures complémentaires. D'une part, il établit une stricte séparation et une relation d'échange fonctionnel entre les agents politiques et les agents économiques: les gardiens sont distingués des producteurs, avec ce double objectif que les premiers sont dans l'incapacité de s'enrichir et les seconds 
d'exercer le pouvoir, et que les producteurs fournissent leur « salaire » ou leur «nourriture » aux gardiens qui, en retour, leur apportent le « salut » politique (V, 463a-b). D'autre part, Socrate place la vie des gardiens sous le signe d'une entière communauté des biens et des personnes (III, 416d417b; IV, 423e-424a; V, 464b-e ; VIII, 543b). Il ne s'agit pas, on le voit, de ne pas faire cas de l'économie, mais au contraire de lui assigner son juste lieu dans la cité pour qu'elle travaille à son unité et à sa communauté plutôt que contre elles. Ce n'est que de cette façon que la cité se « rapproche le plus d'un homme unique » $(\mathrm{V}, 462 \mathrm{c})$, et devient ce lieu entièrement commun où tous les citoyens « autant que possible, peuvent se réjouir et s'affliger pareillement aux mêmes succès comme aux même désastres » $(\mathrm{V}$, $462 b)$.

\section{2) Le Politique}

C'est cependant dans le Politique que la distinction conceptuelle entre l'économique et le politique est la plus poussée, car ce dialogue les envisage dans leur nature propre, et pas seulement par rapport à l'articulation du privé et du commun. Cette distinction s'enracine en effet dans la question de savoir qui fait vraiment la cité, les acteurs économiques étant des prétendants apparemment légitimes à ce titre, et de sérieux concurrents du politique véritable. C'est pourquoi, pour chercher avec son interlocuteur la définition du politique puis de la politique, l'Étranger procède par l'identification successive des candidats potentiels au titre de politique, qu'il disqualifie les uns après les autres jusqu'à ce qu'il trouve le véritable politique. $\mathrm{Au}$ cours de cette démarche, la sphère économique apparaît dans deux passages.

Le premier passage est celui où est appliquée à la politique la distinction entre les causes et les causes auxiliaires, distinction élaborée à propos de l'art du tissage qui sert de paradigme à la politique :

L'Étranger: Tous les arts qui ne fabriquent pas la chose elle-même, mais fournissent à ceux qui la fabriquent des instruments, sans lesquels la tâche propre à chacun de ces arts ne saurait jamais être accomplie, ceux-là sont des causes auxiliaires (ouvaltious) tandis que ceux qui produisent la chose même sont des causes (aitias).

Politique 281e1-3 (trad. L. Brisson, J.-F. Pradeau modifiée).

Appliquée à la cité, cette distinction aboutit à ranger parmi les causes auxiliaires tout ce sans quoi la cité ne pourrait exister mais qui ne la fait pas véritablement - objets et activités d'un côté, techniques ou arts correspondants de l'autre -, et à réserver à la politique le titre de cause véritable de la cité (Pol. 287d1-3). Causes nécessaires mais non suffisantes de la cité, ces auxiliaires sont « les possessions » ( $\kappa \tau \mu \dot{\alpha} \tau \omega \nu$, Pol. 287e1), au sens large 
de choses qu'on trouve dans la cité : elles relèvent de ce qu'on appellerait aujourd'hui le secteur économique. Le passage en question (Pol. 287c7289c3), trop long pour être cité ici, énumère ces possessions et les arts qui leur correspondent ${ }^{31}$. Après la première catégorie, celle des «instruments », à laquelle ne correspond aucun art en propre, la liste se poursuit ainsi : les récipients et l'art de fabriquer les vases; les véhicules, et les arts du charpentier, du potier et du forgeron ; les vêtements, les abris de pierre ou de terre, les armes, les murs, et les arts du tisserand et de l'architecte; le divertissement, et les arts de l'ornementation et de la peinture ; l'or, l'argent, les minerais, les pièces de bois, les peaux animales, les fibres végétales, le liège, le papyrus, les liens, les objets qu'englobe l'espèce « première-née » (soit les matières premières prêtes à l'usage), et les arts de la coupe du bois, du décorticage des matériaux, de l'extraction minière; l'entretien et la nourriture du corps et de ses parties, et les arts de l'agriculture, de la chasse, de la médecine, de la cuisine, de la gymnastique.

Ce passage dessine à grands traits une anthropologie de l'homo faber et de l'homo xeconomicus en ramenant à quelques fonctions élémentaires les opérations ou produits réalisés grâce à la technique et à l'art, et en rassemblant en quelques catégories la multitude d'objets nécessaires au quotidien $^{32}$, pour mieux isoler ensuite l'art politique et son œuvre propre. L'Étranger prend soin de préciser presque à chaque fois que la fonction de l'art correspondant à l'objet ou à l'activité mentionnés ne relève pas de la politique, car certaines proximités terminologiques pourraient laisser penser à une identité fonctionnelle entre la politique et ces arts auxiliaires relevant de la sphère économique. Ces arts assurant la fonction en question au sens propre du terme, ils pourraient en effet revendiquer le titre de cause véritable de la cité - donc prétendre la gouverner - au motif que le politique n'assure cette même fonction que de façon métaphorique. Prenons

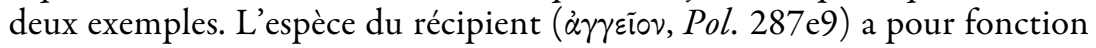
la conservation ou la « sauvegarde » ( $\sigma \omega \tau \eta p i \alpha \varsigma$, Pol. 287e6) de divers pro-

31. Ce passage serait à comparer avec le texte de l'Économique de Xénophon dans lequel Ischomaque décrit à sa femme le rangement très rigoureux d'un bateau phénicien (VIII 11 16), fait l'éloge de l'ordre (VIII 17-23), et décrit comment agencer les objets dans l'oikos (IX 1-10). La classification platonicienne des possessions ne prétend pas être une taxinomie rigoureuse mais un recensement général et ordonné selon des fonctions dont la politique doit être écartée. Cette attention de l'Étranger aux réalités matérielles les plus prosaïques souligne qu'il est possible d'introduire un ordre pratique dans la multiplicité sensible, sans qu'il soit utile - à supposer que cela soit possible - de la circonscrire entièrement (ce que suggère $\sigma \chi \varepsilon \delta \grave{v} v$, Pol. 289a7).

32. Socrate évoque cette multiplicité d'objets au livre II de la République et l'enracine dans ce fait anthropologique que les hommes ont « beaucoup de besoins » $(\pi \circ \lambda \lambda \tilde{\omega} \nu \delta \varepsilon \dot{0}-$ $\mu \varepsilon v o 1$, Rép. II, 369c2). 
duits. Or telle est justement la fonction dévolue aux hommes et aux institutions politiques dans tous les Dialogues: dans la République, les gar-

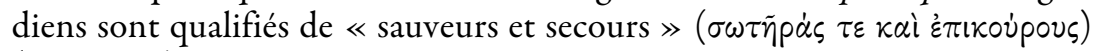
(V, 463b1) et l'éducation droite doit former des hommes qui soient les sauveurs (oi $\sigma \omega \tau \tilde{\eta} \rho \varepsilon \varsigma$, VI, 502d1) du régime politique. De même, la bonne mesure entre richesse et pauvreté, déterminée par le politique, doit garantir la conservation ( $\sigma \omega \tau \eta$ pias) de la cité (Lois, V, 736e4). Ainsi s'explique la fin de la réplique de l'Étranger dans ce passage du Politique : «Cette espèce très variée [...] que nous désignons par cette appellation unique de "récipient", cette espèce couvrant assurément un très vaste domaine, ne convient absolument pas, je crois, à la science recherchée [i.e. la poli-

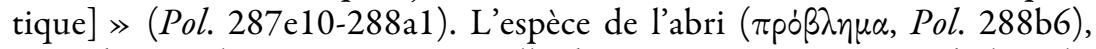
second exemple, permet quant à elle d'écarter une conception de la politique comme protectrice ou défensive : en termes modernes, sa tâche n'est pas d'assurer la sécurité des biens et des personnes en construisant des remparts ou des armes. Ce sont, pour Platon, l'organisation interne de la cité et la paix civile qui garantissent politiquement la sécurité. C'est pourquoi, conclut l'Étranger, « il serait bien plus correct à tous égards de considérer que la plupart [des abris] sont l'ouvrage de l'art de la construction de bâtisses et du tissage plutôt que de la politique » (Pol.288b6-7 ; je souligne).

Le second passage du Politique articulant le politique et l'économique identifie le groupe des subordonnés du politique, dont certains sont des agents économiques : esclaves, commerçants et salariés (Pol. 289c4-290a7). L'Étranger classe ces subordonnés selon le degré croissant de leur revendication possible pour la réalisation de la cité. Ils ne prétendent en effet pas tous à la politique au même degré. Commençons par les esclaves, qui y prétendent « très peu $\gg^{33}$. Leur revendication est sans doute fondée sur le fait que, quelle que soit leur tâche, ce sont bien eux qui l'exécutent, et non leurs maîtres. Ils agissent donc plus directement et plus immédiatement que ces derniers dans la cité, pour autant qu'on considère celle-ci uniquement dans sa réalité matérielle. En précisant qu'on les possède en les achetant, l'Étranger expliquerait pourquoi ces esclaves ne peuvent revendiquer que « très peu » le titre de politique : peut-être est-ce là une référence à ce que

33. Contrairement aux traducteurs qui donnent un sens négatif à $\eta ॅ \kappa \iota \tau \alpha ~(P o l .289 \mathrm{e} 1)$ et traduisent par « ils ne prennent pas part [ou ne prétendent pas le moins du monde] à l'art royal » (Diès 1935, p. 53 ; Brisson et Pradeau 2003, p. 156), il faut donner à ce terme le sens positif de «très peu » : car si les esclaves ne manifestaient aucune prétention à la politique, pourquoi faudrait-il donc les ranger parmi les subordonnés qui sont précisément définis

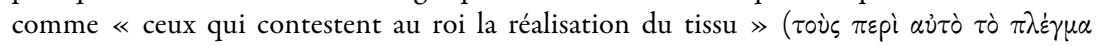

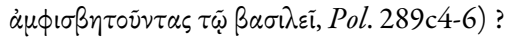


les historiens ont nommé la catégorie de l'esclave « marchandise », dont le statut juridique les prive quasiment de toute prétention politique ${ }^{34}$.

Le degré de revendication augmente avec les commerçants, décrits plus longuement que les esclaves. Platon multiplie à leur sujet les termes connotant l'échange et le mouvement (Pol. 289e4-290a2), sans doute parce que, à l'instar du tisserand réunissant en un même tissu des fils de natures différentes, et du politique réalisant l'unification de la cité à partir de naturels doux et de naturels vifs, les commerçants mettent eux aussi en relation des individus distincts dont ils égalisent les rapports par la transaction commerciale. Forts de cette égalité arithmétique qu'ils réalisent, et conscients qu'ils sont absolument nécessaires pour pourvoir aux besoins des membres de la cité, les commerçants pensent introduire dans la cité une forme de justice que le politique est peut-être impuissant à réaliser. À leurs yeux, le commerce pourrait donc tenir lieu de politique tout court. Contre une telle prétention, l'Étranger et Socrate le Jeune affirment ici clairement que l'échange commercial ne saurait se substituer au véritable lien politique. Celui-ci ne se réduit pas à l'égalité arithmétique de la transaction marchande, quand bien même celle-ci tisse des liens dans les cités.

Enfin, les salariés et les hommes de peine, ces «thètes » situés au plus bas de l'échelle sociale, proches de la servitude mais dont Achille préferrerait toutefois partager le sort plutôt que d'être le roi du pays des morts ${ }^{35}$, sont exclus d'emblée de la course au titre politique : car ce qu'ils vendent, c'est essentiellement leur force physique ${ }^{36}$. Or d'après la première division $\mathrm{du}$ Politique, le politique gouverne moins par la force de son corps que par celle de son âme (Pol.259c6-9). S'il faut néanmoins prendre la peine de les écarter, c'est parce qu'eux aussi participent directement à la réalisation matérielle de la cité.

En résumé, l'objet de ces passages du Politique est de montrer que l'efficience ou la causalité politique n'est pas de même nature que l'efficience ou la causalité économique, et que l'ordre économique doit être subordonné au politique pour que celui-ci accomplisse ce qui lui revient par nature :

L'Étranger : La science qui est réellement royale ne doit pas agir elle-même

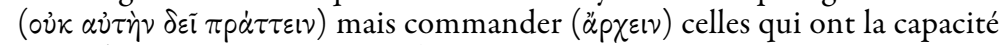
d'agir $(\tau \omega \nu \delta \nu \nu \alpha \mu \dot{\varepsilon} \nu \omega \nu \pi \rho \dot{\alpha} \tau \tau \varepsilon v)$, puisqu'elle discerne, quant à l'opportunité et a l'inopportunité, le départ et l'impulsion des affaires les plus importantes dans les cités, tandis que les autres doivent exécuter ce qu'elle a édicté. (Politique, 305d1-3 (trad. L. Brisson, J.-F. Pradeau modifiée.)

34. Garlan 1982, p. 30-89.

35. Odyssée, X, 488-491.

36. Voir aussi Rép. II, 371e. 
Platon ne considère pas la politique comme une science pratique ou productive - aspects relevant de l'économique - mais comme une science du commandement et de l'usage. Ce qui vaut aà la politique sa suprématie fonctionnelle, c'est qu'elle est l'art de l'usage des arts qui sont compris dans la cité : non pas de l'usage technique de chaque chose ou de chaque objet produit par chaque art de la sphère économique, mais de l'opportunité de l'usage de ces arts par rapport à la finalité de la politique, qui est de réaliser l'unité de la cité par l'entrelacement des caractères humains. La politique sait quel est le bon usage de la cité, et c'est parce qu'elle est cette connaissance qu'elle peut imprimer leur direction aux autres arts, en particulier à ceux qui relèvent du secteur économique ${ }^{37}$. Dans leurs grandes lignes comme dans leurs détails, les projets de cité juste de la République et plus encore des Lois mettent en place des mécanismes et des institutions qui, tout en confirmant la différence de l'économique et du politique, les articulent en donnant à l'économique un rôle de premier plan dans la réalisation du politique et en garantissant à ce dernier son autonomie. Prenons pour exemple les remarques de Platon sur le prêt à intérêt. Dans l'analyse de la division économique croissante des régimes dans la République, Socrate constate que l'oligarchie se caractérise par l'usage répandu du prêt à intérêt. L'usage de cet instrument dans un régime faisant de la richesse la valeur suprême aboutit à l'endettement et à l'appauvrissement des uns, à l'enrichissement des autres, ainsi qu'à une grande instabilité politique :

Socrate : Ceux qui commandent dans cette constitution politique n'exercent leur commandement, je pense, que parce qu'ils ont beaucoup acquis ; ils ne cherchent pas à contrôler les jeunes qui deviennent indisciplinés, pour les empêcher de dépenser leurs biens et leur éviter la ruine. Leur but est, en achetant leurs biens et en leur prêtant à intérêt ( $\varepsilon i \sigma \delta \alpha \nu \varepsilon i \zeta o v \tau \varepsilon \varsigma)$, de devenir encore plus riches et plus considérés. [...] Ainsi dans les oligarchies, c'est en les négligeant et en tolérant l'indiscipline que les dirigeants réduisent parfois à la pauvreté des hommes qui n'étaient pas dépourvus de qualités par leur naissance.

Adimante : C'est certain.

Socrate : Dès lors ces hommes demeurent inactifs dans la cité, où ils sont, je pense, armés de leur aiguillon, les uns criblés de dettes, les autres couverts d'infamie, d'autres subissant l'un et l'autre malheur à la fois ; remplis de haine, ils complotent contre ceux qui se sont appropriés leurs biens et contre tout le monde, désireux d'une seule chose : voir apparaître un régime nouveau.

République, VIII, 555c-e.

Face à cette situation, le remède proposé par Socrate est politique dans son principe. Il consiste en une réforme du prêt à intérêt au moyen d'une 
loi prescrivant « de conclure la plupart des contrats volontaires aux risques du prêteur » (556a-b). Seraient ainsi évités les enrichissements sans scrupules que les plus riches réalisent au détriment des plus pauvres, qu'ils rendent plus pauvres encore (VIII, 556c8-e2). Les Lois vont dans le même sens, mais radicalisent la proposition de la République: l'Athénien propose d'interdire le prêt à intérêt (V, 742c4-6), et d'opposer une fin de non-recevoir à toute plainte qui serait déposée pour non-remboursement d'un prêt sans intérêt (XI, 916d6-e6). Ce retrait de la loi dans les affaires de ce genre est bénéfique de deux façons pour l'unité de la cité: ou bien il diminue la pratique du prêt, évitant ainsi les injustices et les conflits qui pourraient naître de dettes non remboursées ; ou bien, dans le meilleur des cas, il oblige à contracter les prêts sur la base de la seule confiance entre citoyens, à la faveur de sa plus grande cohésion. Dans la République comme dans les Lois, le prêt n'est donc pas interdit, le champ économique n'est pas privé de ses instruments. Qu'il soit autorisé à intérêt dans la République, ou sans dans les Lois, il est, dans les deux cas, encadré par la loi de manière à ne pas entamer l'unité de la cité, voire à la renforcer. C'est là un exemple de l'enracinement à la fois moral et politique que Platon cherche à donner à l'économie pour la mettre au service de l'unité de la cités ${ }^{38}$.

\section{Conclusion}

En 1995, C. Natali appelait de ses vœux un renouveau des perspectives critiques sur l'oikonomia ancienne pour mieux cerner la nature de la pensée économique antique ${ }^{39}$. Cet article prétend avoir fait un pas en ce sens en montrant, d'une part, que cette pensée ne se limite pas aux traités Oikonomike ou Peri oikonomias mais qu'elle se trouve aussi dans des textes consacrés à des questions plus largement politiques; et, d'autre part, que le politique est presque toujours présent même dans ces traités en apparence limités à l'économie domestique, sous la forme d'une réflexion sur les rapports entre l'oikos et la polis ou entre la sphère économique et la sphère politique. S'il est juste et nécessaire de reconnaître que, contrairement à la typologie wébérienne, tout Grec était à la fois homo oconomicus dans l'oikos et homo politicus dans la polis ${ }^{40}$, il semble tout aussi nécessaire de reconnaître, à en juger par les textes que nous avons présentés, que cette double appartenance ne signifiait pas une séparation nette du politique et de l'économique mais une source de questionnement sur la nature complexe de leurs rapports.

38. Un autre exemple serait le rôle du kleros dans les Lois, ainsi que de la politisation des femmes mise en œuvre dans la République comme dans les Lois. Pour des analyses détaillées, voir Helmer 2010, p. 167-266.

39. Natali 1995.

40. Leshem 2013, p. 43-61, en particulier p. 46. 


\section{BIBLIOGRAPHIE}

ARENDT, H. 1961 : Condition de Phomme moderne, Paris, 1961 (Agora, 24).

Brendan Nagle, D. 2006: The Household as the Foundation of Aristotle's polis, Cambridge, 2006.

Brisson, L. et J.-F. Pradeau, 2003 (trad.) : Platon, Le Politique, présentation, traduction et notes, Paris, 2003 (GF, 1156).

Brown, E. 2009: « Politics and Society », dans J. Warren (éd.), The Cambridge Companion to Epicureanism, Cambridge, 2009, p. 179-196 (Cambridge Companions to Philosophy).

Chantraine, P. 2008 (éd. et trad.) : Xénophon, Économique, Introduction et notes revues et complétées par Cl. Mossé, Paris, 2008 (Classiques en poche, 86).

Delattre, D. et V. Tsouna, 2010 (trad.) : Philodème de Gadara. L'Économie (Les Vices, IX), dans D. Delattre et J. Pigeaud (dir.), Les Épicuriens, Paris, 2010 (Bibliothèque de la Pléiade, 564), p. 595-616.

DelatTE, A. 1922 : Essai sur la politique pythagoricienne, Paris, 1922.

DESCAT, R. 1988 : « Aux origines de loikonomia grecque », Quaderni Urbinati di Cultura Classica, New Series, 28/1 (1988), p. 103-119.

DESCAT, R. 2010 : «Thucydide et l'économie, aux origines du logos oikonomikos », dans V. Fromentin, S. Gotteland et P. Payen (éd.), Ombres de Thucydide : la réception de l'historien depuis l'Antiquité jusqu'au début du XXe siècle, Bordeaux-Paris, 2010, p. 403-409.

DIÈs, A. 1935 : Platon, Le Politique, dans CEuvres Complètes, T. IX, 1, Paris, 1935 (CUF, 78).

Dorion, L.-A. (trad.) et M. BANDini, 2000-2011 (éd.) : Xénophon, Les Mémorables, Paris, 2000-2011 (CUF, 399 ; 477 ; 478).

Garlan, Y. 1982 : Les esclaves en Grèce ancienne, Paris, 1982 (Textes à l'appui. Histoire classique).

Gauthier, P. 1976: Un commentaire historique des Poroi de Xénophon, Genève-Paris, 1976 (Hautes études du monde gréco-romain, 8).

Helmer, É. 2010 : La part du bronze : Platon et l'économie, Paris, 2010.

Hense, O. et C. WachSMUTH, 1909 (éd.) : Stobée, Anthologium, Berlin, 1909.

LESHEM, D. 2012 : «The ancient art of economics », The European Journal of the History of Economic Thought, 21/2 (2012), p. 201-229.

LESHEM, D. 2013 : «Oikonomia redefined », Journal of the History of Economic Thought, 35/1 (2013), p. 43-61.

MOREL, P.-M. 2007 : «Les communautés humaines », dans A. Gigandet et P.-M. Morel (éd.), Lire Épicure et les épicuriens, Paris, 2007, p. 167-186 (Quadrige. Manuels).

MULLACH, F. 1867 (éd.) : Fragmenta philosophorum gracorum, vol. II, Paris, 1867.

Natali, C. 1995: «Oikonomia in Hellenistic political thought», dans A. Laks, et M. Schofield, (éd.), Justice and Generosity : Studies in Hellenistic Social and Political Philosophy. Proceedings of the Sixth Symposium Hellenisticum, Cambridge, 1995, p. 95-128.

Pellegrin, P. 1993 (trad.) : Aristote, Les Politiques, traduction inédite, introduction, bibliographie, notes et index, Paris, 1993 (GF, 490).

PLÁCIDO SUÁREZ, D. 2001 : Index thématique des références à l'esclavage et à la dépendance. 8, La dépendance dans lÉconomique de Xénophon, Besançon-Paris, 2001 (Esclavage et dépendance).

Reger, G. 2005 : « The Economy », dans A. Erskine (éd.), A Companion to the Hellenistic World, Malden, 2005 (Blackwell Companions to the Ancient World. Ancient History), p. 331-353. 
Roth, G. et C. WitTich, 1978 (éd. et trad.) : Max Weber, Economy and Society: an Outline of Interpretive Sociology, New York, 1978. [Traduction anglaise de Wirtschaft und Gesellschaft. Grundriss der verstehenden Soziologie, $4^{c}$ éd.]

SCHEIDEL, W., I. MORRIS, R. SALLER, 2007 (éd.) : The Cambridge Economic History of the Greco-Roman World, Cambridge, 2007.

TAlbOT, E. 1859a (trad.) : Xénophon, Économique, Paris, 1859.

TALBOT, E. 1859b (trad.) : Xénophon, Les Revenus, Paris, 1859.

THESLEFF, H. 1965 (éd. et trad.) : The Pythagorean texts of the Hellenistic period, Åbo, 1965.

Wartelle, A. 1968 (trad.): Ps.-Aristote, Économique, Paris, 1968 (CUF, 185). 